\title{
IMPLICIT SUMMATION FORMULA FOR 2-VARIABLE LAGUERRE-BASED POLY-GENOCCHI POLYNOMIALS
}

\author{
WASEEM A. KHAN*, IDREES A. KHAN AND MOIN AHMAD \\ Department of Mathematics, Faculty of Science, Integral University, Lucknow-226026, India \\ *Corresponding author: waseem08_khan@rediffmail.com

\begin{abstract}
The main object of this paper is to introduce a new class of Laguerre-based poly-Genocchi polynomials and investigate some properties for these polynomials and related to the Stirling numbers of the second kind. We derive summation formulae and general symmetry identities by using different analytical means and applying generating functions.
\end{abstract}

\section{INTRODUCTION}

The generalized Bernoulli, Euler and Genocchi polynomials of (real or complex) order $\alpha$ are usually defined by means of the following generating functions (see [1-16]):

$$
\begin{gathered}
\left(\frac{t}{e^{t}-1}\right)^{\alpha} e^{x t}=\sum_{n=0}^{\infty} B_{n}^{(\alpha)}(x) \frac{t^{n}}{n !},\left(|t|<2 \pi ; 1^{\alpha}=1\right), \\
\left(\frac{2}{e^{t}+1}\right)^{\alpha} e^{x t}=\sum_{n=0}^{\infty} E_{n}^{(\alpha)}(x) \frac{t^{n}}{n !},\left(|t|<\pi ; 1^{\alpha}=1\right)
\end{gathered}
$$

and

$$
\left(\frac{2 t}{e^{t}+1}\right)^{\alpha} e^{x t}=\sum_{n=0}^{\infty} G_{n}^{(\alpha)}(x) \frac{t^{n}}{n !},\left(|t|<\pi ; 1^{\alpha}=1\right) .
$$

Received 2017-09-19; accepted 2017-12-07; published 2018-11-02.

2010 Mathematics Subject Classification. 33C45, 33C99, 05A10, 05A15.

Key words and phrases. Laguerre polynomials, poly-Genocchi polynomials, Laguerre-based poly-Genocchi polynomials, summation formulae, symmetric identities.

(C) 2018 Authors retain the copyrights of their papers, and all open access articles are distributed under the terms of the Creative Commons Attribution License. 
So that obviously

$$
B_{n}(x)=B_{n}^{1}(x), E_{n}(x)=E_{n}^{1}(x) \text { and } G_{n}(x)=G_{n}^{1}(x),(n \in \mathbb{N})
$$

where $\mathbb{N}_{0}=\mathbb{N} \cup\{0\}(\mathbb{N}=1,2,3, \cdots)$.

The classical polylogarithmic function $\operatorname{Li}_{k}(z)$ is defined by (see [2], [10]):

$$
\operatorname{Li}_{k}(z)=\sum_{m=1}^{\infty} \frac{z^{m}}{m^{k}},(k \in \mathbb{Z}) .
$$

The poly-Bernoulli numbers and polynomials are defined by following generating functions (see [7], [8], $[9])$ :

$$
\begin{gathered}
\frac{\operatorname{Li}_{k}\left(1-e^{-t}\right)}{e^{t}-1}=\sum_{n=0}^{\infty} B_{n}^{(k)} \frac{t^{n}}{n !} \\
\frac{\operatorname{Li}_{k}\left(1-e^{-t}\right)}{e^{t}-1} e^{x t}=\sum_{n=0}^{\infty} B_{n}^{(k)}(x) \frac{t^{n}}{n !} .
\end{gathered}
$$

In the case $k=1$ in $(1.5)$ and (1.6), we have

$$
B_{n}^{(1)}=B_{n}, B_{n}^{(1)}(x)=B_{n}(x)
$$

The poly-Genocchi numbers and polynomials are defined by following generating functions (see [14]):

$$
\begin{gathered}
\frac{2 \operatorname{Li}_{k}\left(1-e^{-t}\right)}{e^{t}+1}=\sum_{n=0}^{\infty} G_{n}^{(k)} \frac{t^{n}}{n !}, \\
\frac{2 \operatorname{Li}_{k}\left(1-e^{-t}\right)}{e^{t}+1} e^{x t}=\sum_{n=0}^{\infty} G_{n}^{(k)}(x) \frac{t^{n}}{n !} .
\end{gathered}
$$

In the case $k=1$ in $(1.7)$ and (1.8), we have

$$
G_{n}^{(1)}=G_{n}, G_{n}^{(1)}(x)=G_{n}(x)
$$

The 2-variable Laguerre polynomials $(2-\mathrm{VLP}) \mathrm{Ł}_{n}(x, y)$, which is defined by (see [5]):

$$
\frac{1}{(1-y t)} \exp \left(\frac{-x t}{1-y t}\right)=\sum_{n=0}^{\infty} \mathrm{七}_{n}(x, y) t^{n},(|y t|<1)
$$

It is equivalently given by (see [6]).

$$
\exp (y t) C_{0}(x t)=\sum_{n=0}^{\infty} \mathrm{Ł}_{n}(x, y) \frac{t^{n}}{n !},
$$

where $C_{0}(x)$ denotes the $0^{t h}$ order Tricomi function. The $n^{t h}$ order Tricomi functions $C_{n}(x)$ are defined as:

$$
C_{n}(x)=\sum_{r=0}^{\infty} \frac{(-1)^{r} x^{r}}{r !(n+r) !},\left(n \in \mathbb{N}_{0}\right)
$$


with the following generating function:

$$
\exp \left(t-\frac{x}{t}\right)=\sum_{n=0}^{\infty} C_{n}(x) t^{n}
$$

for $t \neq 0$ and for all finite $\mathrm{x}$.

From (1.9) and (1.10), we get

$$
L_{n}(x, y)=n ! \sum_{s=0}^{n} \frac{(-1)^{s} x^{s} y^{n-s}}{(s !)^{2}(n-s) !}=y^{n} L_{n}(x / y) .
$$

Thus, we have

$$
\mathrm{Ł}_{n}(x, 0)=\frac{(-1)^{n} x^{n}}{n !}, \mathrm{Ł}_{n}(0, y)=y^{n}, \mathrm{Ł}_{n}(x, 1)=\mathrm{Ł}_{n}(x),
$$

where $L_{n}(x)$ are the classical Laguerre polynomials (see [1]).

Now, we recall here the following definition as follows:

The Stirling number of the first kind is given by

$$
(x)_{n}=x(x-1) \cdots(x-n+1)=\sum_{l=0}^{n} S_{1}(n, l) x^{l},(n \geq 0)
$$

and the Stirling number of the second kind is defined by generating function:

$$
\left(e^{t}-1\right)^{n}=n ! \sum_{l=n}^{\infty} S_{2}(l, n) \frac{t^{l}}{l !} .
$$

\section{2-VAriable Laguerre-Based poly-Genocchi polynomials}

Let $k \in \mathbb{Z}$, we inroduce 2-variable Laguerre-based poly-Genocchi polynomials by the following generating function:

$$
\frac{2 \operatorname{Li}_{k}\left(1-e^{-t}\right)}{e^{t}+1} \exp (y t) C_{0}(x t)=\sum_{n=0}^{\infty}{ }_{L} G_{n}^{(k)}(x, y) \frac{t^{n}}{n !},
$$

so that

$$
{ }_{L} G_{n}^{(k)}(x, y)=\sum_{m=0}^{n}\left(\begin{array}{c}
n \\
m
\end{array}\right) G_{n-m}^{(k)} L_{m}(x, y) .
$$

When $x=y=0,{ }_{L} G_{n}^{(k)}=G_{n}^{(k)}(0,0)$ are called the poly-Genocchi numbers. For $k=1$ in $(2.1)$, we have

$$
\frac{2 \mathrm{Li}_{1}\left(1-e^{-t}\right)}{e^{t}+1} \exp (y t) C_{0}(x t)=\sum_{n=0}^{\infty}{ }_{L} G_{n}(x, y) \frac{t^{n}}{n !},
$$

where ${ }_{L} G_{n}(x, y)$ is Laguerre-based Genocchi polynomials (see [13]).

Thus, we have

$$
{ }_{L} G_{n}^{(k)}(x, y)={ }_{L} G_{n}(x, y),(n \geq 0)
$$


On setting $x=0,(2.1)$ reduces to the known result of Kim et al. [14.,p.Eq.(4)4776]:

$$
\frac{2 \mathrm{Li}_{k}\left(1-e^{-t}\right)}{e^{t}+1} \exp (y t)=\sum_{n=0}^{\infty} G_{n}^{(k)}(y) \frac{t^{n}}{n !},(k \in \mathbb{Z})
$$

Theorem 2.1. The following explicit summation formulae for Laguerre-based poly-Genocchi polynomials holds true:

$$
{ }_{L} G_{n}^{(2)}(x, y)=\sum_{m=0}^{n}\left(\begin{array}{c}
n \\
m
\end{array}\right) \frac{B_{m} m !}{m+1}{ }_{L} G_{n-m}(x, y) .
$$

Proof. Using generating function for Laguerre-based poly-Genocchi polynomials (2.1), we have

$$
\begin{gathered}
\sum_{n=0}^{\infty}{ }_{L} G_{n}^{(k)}(x, y) \frac{t^{n}}{n !}=\frac{2 \operatorname{Li}_{k}\left(1-e^{-t}\right)}{e^{t}+1} \exp (y t) C_{0}(x t) \\
=\frac{2}{e^{t}+1} \exp (y t) C_{0}(x t) \int_{0}^{t} \frac{1}{e^{z}-1} \int_{0}^{t} \frac{1}{e^{z}-1} \cdots \frac{1}{e^{z}-1} \int_{0}^{t} \frac{z}{e^{z}-1} d z \cdots d z .
\end{gathered}
$$

In particular $k=2$, we have

$$
\begin{gathered}
\sum_{n=0}^{\infty}{ }_{L} G_{n}^{(2)}(x, y) \frac{t^{n}}{n !}=\frac{2}{e^{t}+1} \\
\exp (y t) C_{0}(x t) \int_{0}^{t} \frac{z}{e^{z}-1} d z=\left(\sum_{m=0}^{\infty} \frac{t^{m} B_{m}}{m+1}\right) \frac{2 t}{e^{t}+1} \exp (y t) C_{0}(x t) \\
=\left(\sum_{m=0}^{\infty} \frac{t^{m} B_{m} m !}{m+1 m !}\right)\left(\sum_{n=0}^{\infty}{ }_{L} G_{n}(x, y) \frac{t^{n}}{n !}\right) .
\end{gathered}
$$

Replacing $n$ by $n-m$ in the r.h.s of above equation, we have

$$
\sum_{n=0}^{\infty}{ }_{L} G_{n}^{(2)}(x, y) \frac{t^{n}}{n !}=\sum_{n=0}^{\infty}\left(\sum_{m=0}^{n}\left(\begin{array}{c}
n \\
m
\end{array}\right) \frac{B_{m} m !}{m+1}{ }_{L} G_{n-m}(x, y)\right) \frac{t^{n}}{n !} .
$$

On equating the coefficients of the like powers of $t$ in both sides, we get (2.5).

Remark 2.1. On setting $x=0$, Theorem (2.1) reduces to the known result of Kim et al. [14.,p. 4777, Theorem (2.1)].

Corollary 2.1. For $n \geq 0$, we have

$$
G_{n}^{(2)}(y)=\sum_{m=0}^{n}\left(\begin{array}{c}
n \\
m
\end{array}\right) \frac{B_{m} m !}{m+1} G_{n-m}(y) .
$$

Theorem 2.2. For $n \geq 1$, the degree of ${ }_{L} G_{n}^{(k)}(x, y)$ is n-1. We have

$$
\frac{{ }_{L} G_{n}^{(k)}(x, y)}{n}=\sum_{m=0}^{n-1}\left(\begin{array}{l}
n-1 \\
m
\end{array}\right) \frac{G_{m+1}^{(k)}}{m+1} L_{n-m-1}(x, y) .
$$

Proof. From (2.1), we have

$$
\sum_{n=0}^{\infty}{ }_{L} G_{n}^{(k)}(x, y) \frac{t^{n}}{n !}=\frac{2 \operatorname{Li}_{k}\left(1-e^{-t}\right)}{1-e^{-t}} \exp (y t) C_{0}(x t)
$$




$$
=\left(\sum_{m=0}^{\infty} G_{m}^{(k)} \frac{t^{m}}{m !}\right)\left(\sum_{n=0}^{\infty} L_{n}(x, y) \frac{t^{n}}{n !}\right)
$$

Replacing $n$ by $n-m$ in above equation and comparing the coefficients of $t^{n}$, we get

$$
{ }_{L} G_{n}^{(k)}(x, y)=\sum_{m=0}^{n}\left(\begin{array}{l}
n \\
m
\end{array}\right) G_{m}^{(k)} L_{n-m}(x, y),(n \geq 0) .
$$

From (2.8), we have

$$
\frac{{ }_{L} G_{n}^{(k)}(x, y)}{n}=\sum_{m=0}^{n-1}\left(\begin{array}{l}
n-1 \\
m
\end{array}\right) \frac{G_{m+1}^{(k)}}{m+1} L_{n-m-1}(x, y),(n \geq 1)
$$

Therefore by (2.9), we obtain the result (2.7).

Remark 2.2. For $x=0$, Theorem (2.2) reduces to the known result of Kim et al. [14.,p. 4778, Theorem $(2.2)]$.

Corollary 2.2. For $n \geq 1$, the degree of $G_{n}^{(k)}(x)$ is n-1. We have

$$
\frac{G_{n}^{(k)}(y)}{n}=\sum_{m=0}^{n-1}\left(\begin{array}{l}
n-1 \\
m
\end{array}\right) \frac{G_{m+1}^{(k)}}{m+1} y^{n-m-1} .
$$

Theorem 2.3. For $n \geq 0$, we have

$$
{ }_{L} G_{n}^{(k)}(x, y)=\sum_{p=0}^{n} \sum_{l=1}^{p+1} \frac{(-1)^{l+p+1} l ! S_{2}(p+1, l)}{l^{k}(p+1)}\left(\begin{array}{l}
n \\
p
\end{array}\right){ }_{L} G_{n-p}(x, y) .
$$

Proof. By using (2.1), we can be written as

$$
\sum_{n=0}^{\infty}{ }_{L} G_{n}^{(k)}(x, y) \frac{t^{n}}{n !}=\left(\frac{\mathrm{Li}_{k}\left(1-e^{-t}\right)}{t}\right)\left(\frac{2 t}{e^{t}+1} \exp (y t) C_{0}(x t)\right) .
$$

Now

$$
\begin{gathered}
\frac{1}{t} \operatorname{Li}_{k}\left(1-e^{-t}\right)=\frac{1}{t} \sum_{l=1}^{\infty} \frac{\left(1-e^{-t}\right)^{l}}{l^{k}}=\frac{1}{t} \sum_{l=1}^{\infty} \frac{(-1)^{l}}{l^{k}}\left(1-e^{-t}\right)^{l} \\
=\frac{1}{t} \sum_{l=1}^{\infty} \frac{(-1)^{l}}{l^{k}} l ! \sum_{p=l}^{\infty}(-1)^{p} S_{2}(p, l) \frac{t^{p}}{p !} \\
=\frac{1}{t} \sum_{p=1}^{\infty} \sum_{l=1}^{p} \frac{(-1)^{l+p}}{l^{k}} l ! S_{2}(p, l) \frac{t^{p}}{p !} \\
=\sum_{p=0}^{\infty}\left(\sum_{l=1}^{p+1} \frac{(-1)^{l+p+1}}{l^{k}} l ! \frac{S_{2}(p+1, l)}{p+1}\right) \frac{t^{p}}{p !} .
\end{gathered}
$$

From equations (2.12) and (2.13), we get

$$
\sum_{n=0}^{\infty}{ }_{L} G_{n}^{(k)}(x, y) \frac{t^{n}}{n !}=\sum_{p=0}^{\infty}\left(\sum_{l=1}^{p+1} \frac{(-1)^{l+p+1}}{l^{k}} l ! \frac{S_{2}(p+1, l)}{p+1} \frac{t^{p}}{p !}\right)\left(\sum_{n=0}^{\infty}{ }_{L} G_{n}(x, y) \frac{t^{n}}{n !}\right)
$$


Replacing $n$ by $n-p$ in the r.h.s. of above equation and comparing the coefficients of $t^{n}$ in both sides, we arrive at the desired result (2.11).

Remark 2.3. For $x=0$, Theorem (2.3) reduces to the known result of Kim et al. [14.,p. 4779, Theorem $(2.3)]$.

Corollary 2.3. For $n \geq 0$, we have

$$
G_{n}^{(k)}(y)=\sum_{p=0}^{n} \sum_{l=1}^{p+1} \frac{(-1)^{l+p+1} l ! S_{2}(p+1, l)}{l^{k}(p+1)}\left(\begin{array}{c}
n \\
p
\end{array}\right) G_{n-p}(y) .
$$

Theorem 2.4. For $n \geq 1$, we have

$$
{ }_{L} G_{n}^{(k)}(x, y+1)+{ }_{L} G_{n}^{(k)}(x, y)=2 \sum_{p=1}^{n} \sum_{l=1}^{p} \frac{(-1)^{l+p}}{l^{k}} l ! S_{2}(p, l)\left(\begin{array}{l}
n \\
p
\end{array}\right) L_{n-p}(x, y) .
$$

Proof. By using definition (2.1), we have

$$
\begin{gathered}
\sum_{n=0}^{\infty}{ }_{L} G_{n}^{(k)}(x, y+1) \frac{t^{n}}{n !}+\sum_{n=0}^{\infty}{ }_{L} G_{n}^{(k)}(x, y) \frac{t^{n}}{n !} \\
=\frac{2 \operatorname{Li}_{k}\left(1-e^{-t}\right)}{e^{t}+1} \exp ((y+1) t) C_{0}(x t)+\frac{2 \operatorname{Li}_{k}\left(1-e^{-t}\right)}{e^{t}+1} \exp (y t) C_{0}(x t) \\
=2 \operatorname{Li}_{k}\left(1-e^{-t}\right) \exp (y) t C_{0}(x t) \\
=\sum_{p=1}^{\infty}\left(2 \sum_{l=1}^{p} \frac{(-1)^{l+p}}{l^{k}} l ! S_{2}(p, l)\right) \frac{t^{p}}{p !} \exp (y t) C_{0}(x t) \\
=\left(\sum_{p=1}^{\infty}\left(2 \sum_{l=1}^{p} \frac{(-1)^{l+p}}{l^{k}} l ! S_{2}(p, l)\right) \frac{t^{p}}{p !}\right)\left(\sum_{n=0}^{\infty} L_{n}(x, y) \frac{t^{n}}{n !}\right) .
\end{gathered}
$$

Replacing $n$ by $n-p$ in the above equation and comparing the coefficients of $t^{n}$ in both sides, we obtain the result $(2.15)$.

Remark 2.4. Taking $x=0$, Theorem 2.4 reduces to the known result of Kim et al. [14.,p. 4780, Theorem $(2.4)]$.

Corollary 2.4. For $n \geq 1$, we have

$$
G_{n}^{(k)}(y+1)+G_{n}^{(k)}(y)=2 \sum_{p=1}^{n} \sum_{l=1}^{p} \frac{(-1)^{l+p}}{l^{k}} l ! S_{2}(p, l)\left(\begin{array}{c}
n \\
p
\end{array}\right) y^{n-p} .
$$

Theorem 2.5. For $d \in \mathbb{N}$ with $d \equiv 1(\bmod 2)$, we have

$$
{ }_{L} G_{n}^{(k)}(x, y)=\sum_{p=0}^{n}\left(\begin{array}{c}
n \\
p
\end{array}\right) d^{n-p-1} \sum_{l=0}^{p+1} \sum_{a=0}^{d-1} \frac{(-1)^{l+p+1} l ! S_{2}(p+1, l)}{l^{k}}(-1)^{a}{ }_{L} G_{n-p}\left(\frac{a+y}{d}, x\right) .
$$


Proof. From equation (2.1), we can be written as

$$
\begin{gathered}
\sum_{n=0}^{\infty}{ }_{L} G_{n}^{(k)}(x, y) \frac{t^{n}}{n !}=\frac{2 \operatorname{Li}_{k}\left(1-e^{-t}\right)}{e^{t}+1} \exp (y t) C_{0}(x t) \\
=\left(\frac{2 \operatorname{Li}_{k}\left(1-e^{-t}\right)}{t}\right)\left(\frac{2 t}{e^{d t}+1} \sum_{a=0}^{d-1}(-1)^{a} \exp ((a+y) t) C_{0}(x t)\right) \\
=\left(\sum_{p=0}^{\infty}\left(\sum_{l=1}^{p+1} \frac{(-1)^{l+p+1}}{l^{k}} l ! \frac{S_{2}(p+1, l)}{p+1}\right) \frac{t^{p}}{p !}\right)\left(\sum_{n=0}^{\infty} d^{n-1} \sum_{a=0}^{d-1}(-1)^{a}{ }_{L} G_{n}\left(\frac{a+y}{d}, x\right) \frac{t^{n}}{n !}\right) .
\end{gathered}
$$

Replacing $n$ by $n-p$ in above equation and comparing the coefficient of $t^{n}$ in both sides, we get (2.17).

Remark 2.5. For $x=0$, Theorem 2.5 reduces to the known result of Kim et al. [14.,p. 4780].

Corollary 5. For $d \in \mathbb{N}$ with $d \equiv 1(\bmod 2)$, we have

$$
G_{n}^{(k)}(y)=\sum_{p=0}^{n}\left(\begin{array}{l}
n \\
p
\end{array}\right) d^{n-p-1} \sum_{l=0}^{p+1} \sum_{a=0}^{d-1} \frac{(-1)^{l+p+1} l ! S_{2}(p+1, l)}{l^{k}}(-1)^{a} G_{n-p}\left(\frac{a+y}{d}\right) .
$$

\section{Summation formulae for Laguerre-Based poly-Genocchi polynomials}

In this section, we establish summation formula for Laguerre-based poly-Genocchi polynomials by using series techniques method.

Theorem 3.1. The following implicit summation formulae for Laguerre-based poly-Genocchi polynomials ${ }_{L} G_{n}^{(k)}(x, y)$ holds true:

$$
{ }_{L} G_{l+p}^{(k)}(x, z)=\sum_{m, n=0}^{l, p}\left(\begin{array}{l}
l \\
m
\end{array}\right)\left(\begin{array}{l}
p \\
n
\end{array}\right)(z-y)^{m+n}{ }_{L} G_{l+p-m-n}^{(k)}(x, y) .
$$

Proof. Replacing $t$ by $t+u$ and rewrite the generating function (2.1) as

$$
\frac{2 \operatorname{Li}_{k}\left(1-(e)^{-(t+u)}\right)}{e^{t+u}+1} C_{0}(x(t+u))=e^{-y(t+u)} \sum_{l, p=0}^{\infty}{ }_{L} G_{l+p}^{(k)}(x, y) \frac{t^{l}}{l !} \frac{u^{p}}{p !} .
$$

Replacing $y$ by $z$ in the above equation and equating the resulting equation to the above equation, we get

$$
e^{(z-y)(t+u)} \sum_{m, l=0}^{\infty}{ }_{L} G_{l+p}^{(k)}(x, y) \frac{t^{l}}{l !} \frac{u^{p}}{p !}=\sum_{l, p=0}^{\infty}{ }_{L} G_{l+p}^{(k)}(x, z) \frac{t^{l}}{l !} \frac{u^{p}}{p !} .
$$

On expanding exponential function (3.3) gives

$$
\sum_{N=0}^{\infty} \frac{[(z-y)(t+u)]^{N}}{N !} \sum_{l, p=0}^{\infty}{ }_{L} G_{l+p}^{(k)}(x, y) \frac{t^{l}}{l !} \frac{u^{p}}{p !}=\sum_{l, p=0}^{\infty}{ }_{L} G_{l+p}^{(k)}(x, z) \frac{t^{l}}{l !} \frac{u^{p}}{p !}
$$

which on using formula [16, p.52(2)]

$$
\sum_{N=0}^{\infty} f(N) \frac{(x+y)^{N}}{N !}=\sum_{n, m=0}^{\infty} f(n+m) \frac{x^{n}}{n !} \frac{y^{m}}{m !}
$$


in the left hand side becomes

$$
\sum_{m, n=0}^{\infty} \frac{(z-x)^{m+n} t^{m} u^{n}}{m ! n !} \sum_{l, p=0}^{\infty}{ }_{H} G_{l+p}^{(k)}(x, y) \frac{t^{l}}{l !} \frac{u^{p}}{p !}=\sum_{l, p=0}^{\infty}{ }_{H} G_{l+p}^{(k)}(z, y) \frac{t^{l}}{l !} \frac{u^{p}}{p !}
$$

Now replacing $l$ by $l-m, p$ by $p-n$ and using the lemma [16, p.100(1)] in the left hand side of (3.6), we get

$$
\begin{gathered}
\sum_{m, n=0}^{\infty} \sum_{l, p=0}^{\infty} \frac{(z-x)^{m+n}}{m ! n !}{ }_{L} G_{l+p-m-n}^{(k)}(x, y) \frac{t^{l}}{(l-m) !} \frac{u^{p}}{(p-n) !} \\
=\sum_{l, p=0}^{\infty}{ }_{L} G_{l+p}^{(k)}(x, z) \frac{t^{l}}{l !} \frac{u^{p}}{p !} .
\end{gathered}
$$

Finally on equating the coefficients of the like powers of $t$ and $u$ in the above equation, we get the required result.

Remark 3.1. Taking $l=0$ in assertion (3.1) of Theorem 3.1, we deduce the following consequence of Theorem 3.1 .

Corollary 3.1. The following summation formula for Laguerre-based poly-Genocchi polynomials ${ }_{H} G_{n}^{(k)}(z, y)$ holds true:

$$
{ }_{L} G_{p}^{(k)}(x, z)=\sum_{n=0}^{p}\left(\begin{array}{c}
p \\
n
\end{array}\right)(z-y)^{n}{ }_{L} G_{p-n}^{(k)}(x, y)
$$

Remark 3.2. Replacing $z$ by $z+y$ in (3.8), we obtain

$$
{ }_{L} G_{p}^{(k)}(x, z+y)=\sum_{n=0}^{p}\left(\begin{array}{c}
p \\
n
\end{array}\right) z^{n}{ }_{L} G_{p-n}^{(k)}(x, y)
$$

Theorem 3.2. The following summation formula for Laguerre-based poly-Genocchi polynomials ${ }_{H} G_{n}^{(k)}(z, y)$ holds true:

$$
{ }_{L} G_{n}^{(k)}(x, y+u)=\sum_{j=0}^{n}\left(\begin{array}{c}
n \\
j
\end{array}\right) u^{j}{ }_{L} G_{n-j}^{(k)}(x, y)
$$

Proof. Using (2.1), we can be written as

$$
\sum_{n=0}^{\infty}{ }_{L} G_{n}^{(k)}(x, y+u) \frac{t^{n}}{n !}=\frac{2 \operatorname{Li}_{k}\left(1-e^{-t}\right)}{e^{t}+1} \exp ((y+u) t) C_{0}(x t)=\left(\sum_{n=0}^{\infty}{ }_{L} G_{n}^{(k)}(x, y) \frac{t^{n}}{n !}\right)\left(\sum_{j=0}^{\infty} u^{j} \frac{t^{j}}{j !}\right)
$$

Now replacing $n$ by $n-j$ and comparing the coefficients of $t^{n}$ in both sides, we obtain (3.10). 
Theorem 3.3. The following summation formula for Laguerre-based poly-Genocchi polynomials ${ }_{H} G_{n}^{(k)}(z, y)$ holds true:

$$
{ }_{L} G_{n}^{(k)}(x+w, y+u)=\sum_{m=0}^{n}\left(\begin{array}{c}
n \\
m
\end{array}\right){ }_{L} G_{n-m}^{(k)}(x, y) L_{m}(u, w) .
$$

Proof. From (2.1) and (1.10), we have

$$
\frac{2 \operatorname{Li}_{k}\left(1-(e)^{-t}\right)}{e^{t}+1} \exp ((y+u) t) C_{0}((x+w) t)=\left(\sum_{n=0}^{\infty}{ }_{L} G_{n}^{(k)}(x, y) \frac{t^{n}}{n !}\right)\left(\sum_{m=0}^{\infty} L_{m}(u, w) \frac{t^{m}}{m !}\right) .
$$

Now replacing $n$ by $n-m$ and comparing the coefficients of $t^{n}$ in both sides, we get (3.11).

Theorem 3.4. The following summation formula for Laguerre-based poly-Genocchi polynomials ${ }_{L} G_{n}^{(k)}(x, y)$ holds true:

$$
{ }_{L} G_{n}^{(k)}(x, y+1)=\sum_{m=0}^{n}\left(\begin{array}{l}
n \\
m
\end{array}\right){ }_{L} G_{n-m}^{(k)}(x, y) .
$$

Proof. Using definition (2.1), we have

$$
\begin{aligned}
\sum_{n=0}^{\infty}{ }_{L} G_{n}^{(k)}(x, y+1) \frac{t^{n}}{n !}-\sum_{n=0}^{\infty}{ }_{L} G_{n}^{(k)}(x, y) \frac{t^{n}}{n !}=\frac{2 \operatorname{Li}_{k}\left(1-e^{-t}\right)}{e^{t}+1} \exp (y t) C_{0}(x t)\left(e^{t}-1\right) \\
=\left(\sum_{n=0}^{\infty}{ }_{L} G_{n}^{(k)}(x, y) \frac{t^{n}}{n !}\right)\left(\sum_{m=0}^{\infty} \frac{t^{m}}{m !}\right)-\sum_{n=0}^{\infty}{ }_{L} G_{n}^{(k)}(x, y) \frac{t^{n}}{n !} \\
=\sum_{n=0}^{\infty} \sum_{m=0}^{n}{ }_{L} G_{n-m}^{(k)}(x, y) \frac{t^{n}}{(n-m) ! m !}-\sum_{n=0}^{\infty}{ }_{L} G_{n}^{(k)}(x, y) \frac{t^{n}}{n !} .
\end{aligned}
$$

Finally, equating the coefficients of the like powers of $t^{n}$, we get (3.12).

\section{Identities for 2-VARiable Laguerre-Based poly-Genocchi Polynomials}

In this section, we derive general symmetry identities for 2-variable Laguerre-based poly-Genocchi polynomials ${ }_{L} G_{n}^{(k)}(x, y)$ by applying the generating function(2.1). Such type of identities have been introduced by several authors (see [11], [12], [13], [15]).

Theorem 4.1. Let $a, b>0$ and $a \neq b, x, y \in \mathbb{R}, n \geq 0$, then the following identity holds true:

$$
\begin{aligned}
& \sum_{m=0}^{n}\left(\begin{array}{c}
n \\
m
\end{array}\right) a^{n-m} b_{L}^{m} G_{n-m}^{(k)}(b x, b y)_{L} G_{m}^{(k)}(a u, a w) \\
= & \sum_{m=0}^{n}\left(\begin{array}{l}
n \\
m
\end{array}\right) a^{m} b^{n-m}{ }_{L} G_{n-m}^{(k)}(a x, a y)_{L} G_{m}^{(k)}(b u, b w) .
\end{aligned}
$$


Proof. Let

$$
G(t)=\left(\frac{\left(2 \operatorname{Li}_{k}\left(1-e^{-a t}\right)\left(2 \operatorname{Li}_{k}\left(1-e^{-b t}\right)\right)\right.}{\left(e^{a t}+1\right)\left(A^{b t}-B^{-b t}\right)}\right) \exp (a b(y+u) t) C_{0}(a b x t) C_{0}(a b w t) .
$$

Since $G(t)$ is symmetric in $a$ and $b$ and $G(t)$ can written as

$$
\begin{gathered}
G(t)=\sum_{n=0}^{\infty}{ }_{L} G_{n}^{(k)}(b x, b y) \frac{(a t)^{n}}{n !} \sum_{m=0}^{\infty}{ }_{L} G_{m}^{(k)}(a u, a w) \frac{(b t)^{m}}{m !} \\
G(t)=\sum_{n=0}^{\infty}\left(\sum_{m=0}^{n}\left(\begin{array}{c}
n \\
m
\end{array}\right) a^{n-m} b^{m}{ }_{L} G_{n-m}^{(k)}(b x, b y)_{L} G_{m}^{(k)}(a u, a w)\right) \frac{t^{n}}{n !} .
\end{gathered}
$$

Similarly, we can show that

$$
\begin{gathered}
G(t)=\sum_{n=0}^{\infty}{ }_{L} G_{n}^{(k)}(a x, a y) \frac{(b t)^{n}}{n !} \sum_{m=0}^{\infty}{ }_{L} G_{m}^{(k)}(b u, b w) \frac{(a t)^{m}}{m !} \\
G(t)=\sum_{n=0}^{\infty}\left(\sum_{m=0}^{n}\left(\begin{array}{c}
n \\
m
\end{array}\right) a^{m} b^{n-m}{ }_{L} G_{n-m}^{(k)}(a x, a y)_{L} G_{m}^{(k)}(b u, b w)\right) \frac{t^{n}}{n !} .
\end{gathered}
$$

Comparing the coefficients of $\frac{t^{n}}{n !}$ in (4.3) and (4.4), we arrive at the desired result.

Remark 4.1. On setting $b=1$ in Theorem 4.1, we get

$$
\begin{aligned}
& \sum_{m=0}^{n}\left(\begin{array}{c}
n \\
m
\end{array}\right) a^{n-m}{ }_{L} G_{n-m}^{(k)}(x, y)_{L} G_{m}^{(k)}(a u, a w) \\
& =\sum_{m=0}^{n}\left(\begin{array}{c}
n \\
m
\end{array}\right) a^{m}{ }_{L} G_{n-m}^{(k)}(a x, a y)_{L} G_{m}^{(k)}(u, w) .
\end{aligned}
$$

Theorem 4.2. Let $a, b>0$ and $a \neq b, x, y \in \mathbb{R}$ and $n \geq 0$, then the following identity holds true:

$$
\begin{aligned}
& \sum_{m=0}^{n}\left(\begin{array}{c}
n \\
m
\end{array}\right) \sum_{i=0}^{a-1} \sum_{j=0}^{b-1}{ }_{L} G_{n-m}^{(k)}\left(b y+\frac{b}{a} i+j, b x\right){ }_{L} G_{m}^{(k)}(a u, a w) b^{m} a^{n-m} \\
= & \sum_{m=0}^{n}\left(\begin{array}{c}
n \\
m
\end{array}\right) \sum_{i=0}^{b-1} \sum_{j=0}^{a-1}{ }_{L} G_{n-m}^{(k)}\left(a y+\frac{a}{b} i+j, a x\right){ }_{L} G_{m}^{(k)}(b u, b w) a^{m} b^{n-m} .
\end{aligned}
$$

Proof. Let

$$
\begin{gathered}
G(t)=\left(\frac{\left(2 \operatorname{Li}_{k}\left(1-e^{-a t}\right)\right)\left(2 \operatorname{Li}_{k}\left(1-e^{-b t}\right)\right)}{\left(e^{a t}+1\right)^{2}\left(e^{b t}+1\right)^{2}}\right)\left(e^{a b t}+1\right)^{2} \exp (a b(y+u) t) C_{0}(a b x t) C_{0}(a b w t) \\
G(t)=\left(\frac{2 \operatorname{Li}_{k}\left(1-e^{-a t}\right)}{e^{a t}+1}\right) \exp (a b y t) C_{0}(a b x t)\left(\frac{e^{a b t}+1}{e^{b t}+1}\right)\left(\frac{2 \operatorname{Li}_{k}\left(1-e^{-b t}\right)}{e^{b t}+1}\right) \\
\times \exp (a b u t) C_{0}(a b w t)\left(\frac{e^{a b t}+1}{e^{a t}+1}\right)
\end{gathered}
$$




$$
\begin{gathered}
=\left(\frac{2 \operatorname{Li}_{k}\left(1-e^{-a t}\right)}{\left(e^{a t}+1\right.}\right) \exp (a b y t) C_{0}(a b x t) \sum_{i=0}^{a-1}(-1)^{i} e^{b t i}\left(\frac{2 \operatorname{Li}_{k}\left(1-e^{-b t}\right)}{e^{b t}+1}\right) \\
\times \exp (a b u t) C_{0}(a b w t) \sum_{j=0}^{b-1}(-1)^{j} e^{a t j} \\
=\left(\frac{2 \operatorname{Li}_{k}\left(1-e^{-t}\right)}{e^{a t}+1}\right) C_{0}(a b x t) \sum_{i=0}^{a-1} \sum_{j=0}^{b-1}(-1)^{i+j} e^{\left(b y+\frac{b}{a} i+j\right) a t} \sum_{m=0}^{\infty}{ }_{L} G_{m}^{(k)}(a u, a w) \frac{(b t)^{m}}{m !} \\
=\sum_{n=0}^{\infty} \sum_{i=0}^{a-1} \sum_{j=0}^{b-1}{ }_{L} G_{n}^{(k)}\left(b y+\frac{b}{a} i+j, b x\right) \frac{(a t)^{n}}{n !} \sum_{m=0}^{\infty}{ }_{L} G_{m}^{(k)}(a u, a w) \frac{(b t)^{m}}{(m) !} \\
G(t)=\sum_{n=0}^{\infty}\left(\sum_{m=0}^{n}\left(\begin{array}{c}
n \\
m
\end{array}\right) \sum_{i=0}^{a-1} \sum_{j=0}^{b-1}(-1)^{i+j}{ }_{L} G_{n-m}^{(k)}\left(b y+\frac{b}{a} i+j, b x\right){ }_{L} G_{m}^{(k)}(a u, a w) b^{m} a^{n-m}\right) \frac{t^{n}}{n !} .
\end{gathered}
$$

On the other hand

$$
G(t)=\sum_{n=0}^{\infty}\left(\sum_{m=0}^{n}\left(\begin{array}{l}
n \\
m
\end{array}\right) \sum_{i=0}^{b-1} \sum_{j=0}^{a-1}(-1)^{i+j}{ }_{L} G_{n-m}^{(k)}\left(a y+\frac{a}{b} i+j, a x\right){ }_{L} G_{m}^{(k)}(b u, b w) a^{m} b^{n-m}\right) \frac{t^{n}}{n !} .
$$

On comparing the coefficients of $\frac{t^{n}}{n !}$ in (4.6) and (4.7), we arrive at the desired result (4.5).

Acknowledgement. All authors would like to thank Integral University, Lucknow, India, for providing the manuscript number IU/R\&D/2017-MCN000240 for the present research work.

\section{REFERENCES}

[1] L. C. Andrews, Special functions for engineers and mathematicians, Macmillan Co. New York, 1985.

[2] A. Bayad and Y. Hamahata, Polylogarithms and poly-Bernoulli polynomials, Kyushu. J. Math. 65(2011), 15-24.

[3] C. H. Chang and C. W. Ha, On recurrence relation for Bernoulli and Euler numbers, Bull. Aust. Math. Soc. 64(2001), 469-474.

[4] G. S. Cheon, A note on the Bernoulli and Euler polynomials, App. Math. Lett. 16(3)(2003), 365-368.

[5] G. Dattoli, A. Torre, Operational methods and two variable Laguerre polynomials, Atti Accad. Sci. Torino Cl. Sci. Fis. Mat. Natur. 132(1998) 3-9.

[6] G. Dattoli, A. Torre and A. M. Mancho, The generalized Laguerre polynomials, the associated Bessel functions and applications to propagation problems, Radiat. Phys. Chem. 59(2000), 229-237.

[7] H. Jolany, M. R. Darafsheh, R. E. Alikelaye, Generalizations of Poly-Bernoulli Numbers and Polynomials, Int. J. Math. Comb. 2(2010), 7-14.

[8] H. Jolany, R. B. Corcino, Explicit formula for generalization of Poly-Bernoulli numbers and polynomials with a,b,c parameters, J. Class. Anal. 6(2015), 119-135.

[9] H. Jolany, M. Aliabadi, R. B. Corcino and M. R. Darafsheh, A Note on Multi Poly-Euler Numbers and Bernoulli Polynomials, Gen. Math. 20(2-3)(2012), 122-134.

[10] M. Kaneko, Poly-Bernoulli numbers, J. Thor. Nombres Bordx. 9 (1997), 221-228.

[11] W. A. Khan, Some properties of the generalized Apostol type Hermite-Based polynomials, Kyungpook Math. J. 55(2015), 597-614. 
[12] W. A. Khan, A note on Hermite-based poly-Euler and multi poly-Euler polynomials, Palestine J. Math. 6(2017), $204-214$.

[13] W. A. Khan, S. Araci, M. Acikgoz, A new class of Laguerre-based Apostol type polynomials, Cogent Math. 3(2016), Art. ID 1243839.

[14] T. Kim, Y. S. Jang and J. J. Seo, A note on poly-Genocchi numbers and polynomials, Appl. Math. Sci. 8(2014), $4475-4781$.

[15] M. A. Pathan and W. A. Khan, Some implicit summation formulas and symmetric identities for the generalized HermiteEuler polynomials, East-West J. Math. 16(1) (2014), 92-109.

[16] H. M. Srivastava and H. L. Manocha, A treatise on generating functions Ellis Horwood Limited, New York, 1984. 